\title{
STRONG PSEUDOPRIMES TO THE FIRST EIGHT PRIME BASES
}

\author{
YUPENG JIANG AND YINGPU DENG
}

\begin{abstract}
Define $\psi_{m}$ to be the smallest strong pseudoprime to the first $m$ prime bases. The exact value of $\psi_{m}$ is known for $1 \leq m \leq 8$. Z. Zhang has found a 19-decimal-digit number $Q_{11}=3825123056546413051$ which is a strong pseudoprime to the first 11 prime bases and he conjectured that $\psi_{9}=\psi_{10}=\psi_{11}=Q_{11}$. We tabulate all the strong pseudoprimes $n \leq Q_{11}$ to the first eight prime bases, and prove Zhang's conjecture.
\end{abstract}

\section{INTRODUCTION}

If $n$ is prime, in view of Fermat's Little Theorem, the congruence

$$
a^{n-1} \equiv 1 \quad \bmod n
$$

holds for every $a$ with $n \nmid a$. There are composite numbers also satisfying this congruence. Such an odd composite number $n$ is called a pseudoprime to base $a$ $\left(\operatorname{psp}(a)\right.$ for short). Moreover, for an odd prime $n$, letting $n-1=2^{s} d$ with $d$ odd, we have

$$
a^{d} \equiv 1 \bmod n
$$

or

$$
a^{2^{k} d} \equiv-1 \quad \bmod n
$$

for an integer $k$ with $0 \leq k<s$. If a $\operatorname{psp}(a) n$ satisfies one of these two equations, we call $n$ a strong pseudoprime to base $a(\operatorname{spsp}(a)$ for short). This is the basis of the Miller-Rabin test [5].

Define $\psi_{m}$ to be the smallest strong pseudoprime to each of the first $m$ prime bases. If $n<\psi_{m}$, then only $m$ strong pseudoprime tests are needed to determine whether $n$ is prime or not. If we know the exact value of $\psi_{m}$, then for integers $n<\psi_{m}$, there is a deterministic primality testing algorithm which is easier to understand and also faster than other known tests. The exact value of $\psi_{m}$ for $1 \leq$ $m \leq 8$ is known [2,4]: $\psi_{1}=2047, \psi_{2}=1373653, \psi_{3}=25326001, \psi_{4}=3215031751$, $\psi_{5}=2152302898747, \psi_{6}=3474749660383, \psi_{7}=\psi_{8}=341550071728321$.

Received by the editor August 23, 2012 and in revised form, January 26, 2013 and April 5, 2013.

2010 Mathematics Subject Classification. Primary 11Y11, 11A51.

Key words and phrases. Strong pseudoprimes, Chinese Remainder Theorem.

This research was supported by the NNSF of China (Grant Nos. 11071285, 61121062), 973 Project (2011CB302401) and the National Center for Mathematics and Interdisciplinary Sciences, CAS. 
In [2, Jaeschke also gave upper bounds for $\psi_{9}, \psi_{10}$ and $\psi_{11}$. These bounds were improved by Z. Zhang several times and Zhang conjectured that

$$
\begin{aligned}
\psi_{9}=\psi_{10}=\psi_{11}=Q_{11} & =3825123056546413051 \\
& =149491 \cdot 747451 \cdot 34233211
\end{aligned}
$$

Zhang also determined upper bounds and stated conjectures for $\psi_{m}$, with $12 \leq$ $m \leq 20$ (see $6,8,9$ ).

In this paper, we calculate all the strong pseudoprimes $n \leq Q_{11}$ to the first eight prime bases and obtain the following conclusion.

Theorem 1.1. $\psi_{9}=\psi_{10}=\psi_{11}=3825123056546413051$.

This article is organized in the following way. In Section 2 we give notations and basic facts needed for our algorithm. Just as in 2], we consider the number of prime divisors of the testing number. Let $n=p_{1} p_{2} \cdots p_{t}$. In Section 3 we consider $t \geq 4$, in Section 4, the case $t=3$, and Section 5 , the case $t=2$. In Section 6 we obtain our conclusion, and report the total time required for our algorithm.

\section{Foundations of the ALGORIthm}

In this section, we give the foundations for our algorithm. Let $p$ be a prime and $a$ an integer with $p \nmid a$. Denote the smallest positive integer $e$ such that $a^{e} \equiv 1$ $\bmod p$ by $\operatorname{ord}_{p}(a)$. Moreover, for any integer $n$, if $n=p^{e} n^{\prime}$ with $p \nmid n^{\prime}$ and $e \geq 0$, we denote $e$ by $v_{p}(n)$. In this article, we only use $v_{p}(n)$ for $p=2$, so we write $v(n)$ for this case. For $\nu \in \mathbb{Z}^{m}, \nu=\left(a_{1}, \ldots, a_{m}\right)$ with $p \nmid a_{i}$ for each $i$, we define $\sigma_{p}^{\nu}$ by

$$
\sigma_{p}^{\nu}=\left(v\left(\operatorname{ord}_{p}\left(a_{1}\right)\right), \ldots, v\left(\operatorname{ord}_{p}\left(a_{m}\right)\right)\right) .
$$

If $n$ is a pseudoprime (or strong pseudoprime) for all the $a_{i}$ 's, we denote it by $\operatorname{psp}(\nu)$ $($ or $\operatorname{spsp}(\nu))$.

We need to check all odd integers less than $Q_{11}$ to see if there are strong pseudoprimes to the first eight prime bases. First, we exclude the integers having square divisors. If $n$ is $\operatorname{asp}(a)$ and $p^{2} \mid n$ for some prime $p$, then we have

$$
a^{n-1} \equiv 1 \quad \bmod p^{2} .
$$

Also,

$$
a^{p(p-1)} \equiv 1 \quad \bmod p^{2},
$$

and since $\operatorname{gcd}(p, n-1)=1$, we have

$$
a^{p-1} \equiv 1 \quad \bmod p^{2} .
$$

If $a=2$ and 3 , then

$$
2^{p-1} \equiv 1 \quad \bmod p^{2}, \quad 3^{p-1} \equiv 1 \quad \bmod p^{2} .
$$

These two equations do not hold simultaneously for any prime $p$ less than $3 \cdot 10^{9}$, which is greater than $\sqrt{Q_{11}} \approx 1.9 \cdot 10^{9}$, so we only need to consider squarefree integers. In fact, by a search of Knauer and Richstein 3 , the smallest prime which satisfies these two equations must be greater than $1.25 \cdot 10^{15}$. This bound is improved to $6.7 \cdot 10^{15}$ by Dorais and Klyve[1]. Thus all $\operatorname{psp}(2,3)$ 's less than $4.4 \cdot 10^{31}$ are squarefree.

Now we give the following important proposition (Proposition 1 in [2]). 
Proposition 2.1. Let $n=p_{1} \cdots p_{t}$ with different primes $p_{1}, \ldots, p_{t}$, and $\nu=$ $\left(a_{1}, \ldots, a_{m}\right)$ with different integers such that $p_{j} \nmid a_{i}$ for all $i=1, \ldots, m$ and $j=1, \ldots, t$. Then $n$ is an $\operatorname{spsp}(\nu)$ if and only if $n$ is a $p s p(\nu)$ and $\sigma_{p_{1}}^{\nu}=\cdots=\sigma_{p_{t}}^{\nu}$.

Example 2.2. Consider $\psi_{3}=25326001=p_{1} p_{2}=2251 \cdot 11251$ and $\nu=(2,3,5)$. Then $\psi_{3}$ is an $\operatorname{spsp}(\nu)$. We have

$$
\operatorname{ord}_{p_{1}}(2)=750, \quad \operatorname{ord}_{p_{1}}(3)=250, \quad \operatorname{ord}_{p_{1}}(5)=1125
$$

and

$$
\operatorname{ord}_{p_{2}}(2)=2250, \quad \operatorname{ord}_{p_{2}}(3)=2250, \quad \operatorname{ord}_{p_{2}}(5)=1125 .
$$

Thus, $\sigma_{p_{1}}^{\nu}=\sigma_{p_{2}}^{\nu}=(1,1,0)$.

This is the main necessary condition that we use to find strong pseudoprimes. In our algorithm, $\nu=(2,3,5,7,11,13,17,19)$. For a given prime $p$, we find primes $q$ satisfying $\sigma_{p}^{\nu}=\sigma_{q}^{\nu}$. Moreover, we use the following two propositions to reduce the number of candidates. Let $\left(\frac{a}{p}\right)$ denote the Legendre symbol.

Proposition 2.3. For primes $p$ and $q$, if $v(p-1)=v(q-1)$ and $\sigma_{p}^{(a)}=\sigma_{q}^{(a)}$, then $\left(\frac{a}{p}\right)=\left(\frac{a}{q}\right)$.

Proposition 2.4. For primes $p$ and $q$, if $v(p-1)<v(q-1)$ and $\sigma_{p}^{(a)}=\sigma_{q}^{(a)}$, then $\left(\frac{a}{q}\right)=1$.

The proofs of these two propositions are similar, and Proposition 2.3 is Proposition 3 in [2]. We omit both proofs. The following example explains how we can reduce the number of candidates.

Example 2.5. Assume $p=23$, and we need to find a prime $q$ with $\sigma_{p}^{\nu}=\sigma_{q}^{\nu}$ for $\nu=(2,3)$. First, if $q \equiv 3 \bmod 4$, then by Proposition 2.3 we have $\left(\frac{2}{23}\right)=\left(\frac{2}{q}\right)$ and $\left(\frac{3}{23}\right)=\left(\frac{3}{q}\right)$. Therefore, $q \equiv 7 \bmod 8$ and $q \equiv 2 \bmod 3$. By the Chinese Remainder Theorem, we obtain $q \equiv 23 \bmod 24$. If $q \equiv 1 \bmod 4$, then $\left(\frac{2}{q}\right)=\left(\frac{3}{q}\right)=1$ by Proposition 2.4, so $q \equiv 1 \bmod 8$ and $q \equiv 1 \bmod 3$. By the Chinese Remainder Theorem, $q \equiv 1 \bmod 24$. Thus, $q \equiv \pm 1 \bmod 24$. We only need to check two of the total $\phi(24)=8$ residue classes modulo 24 . In fact, we only check $q \equiv-1 \bmod 24$ in our algorithm. We define binary primes to deal with the $q \equiv 1 \bmod 24$ case. See the details in Section 4.

\section{3. $t \geq 5$ AND $t=4$}

From now on, we fix $\nu=(2,3,5,7,11,13,17,19)$. If $n$ is a $\operatorname{psp}(\nu)$ and prime $p \mid n$, as $a^{n-1} \equiv 1 \bmod p$, then $\operatorname{ord}_{p}(a) \mid(n-1)$, for all $a=2,3,5,7,11,13,17,19$. Define $\lambda_{p}$ to be the least common multiple of the eight orders, i.e.,

$$
\lambda_{p}=\operatorname{lcm}\left\{\operatorname{ord}_{p}(a): a=2,3,5,7,11,13,17,19\right\} .
$$

Then we have $\lambda_{p} \mid(n-1)$. Since we only need to consider squarefree integers, we always denote $n=p_{1} \cdots p_{t}$ with $p_{1}<\cdots<p_{t}$. In this section, we consider two cases: first, $t \geq 5$, and second, $t=4$. 
3.1. $t \geq 5$. For each $p \leq\left[\sqrt[5]{Q_{11}}\right]=5206$, let $S_{p}$ be the set of all primes $q$ with $\sigma_{q}^{\nu}=\sigma_{p}^{\nu}$, and denote the $k$ th largest element in $S_{p}$ by $s_{p, k}$. Our algorithm outputs the first $l$ elements of $S_{p}$ with $l \geq 5$ and

$$
\prod_{i=1}^{5} s_{p, i} \leq Q_{11}, \quad\left(\prod_{i=1}^{4} s_{p, i}\right) s_{p, l} \leq Q_{11}, \quad\left(\prod_{i=1}^{4} s_{p, i}\right) s_{p, l+1}>Q_{11} .
$$

There are 60 such sequences for all $p \leq 5206$ and the algorithm takes less than one minute to find them. We tabulate some sequences in the following table. The sequences are numbered according to the size of their first primes. $l$ is the total number of primes in each sequence. The last column is the number of integers $n=p_{1} p_{2} p_{3} p_{4} p_{5} \leq Q_{11}$ with each $p_{i}$ in this sequence.

TABle 1. Sequences with equal $\sigma_{p}^{\nu}$

\begin{tabular}{|c|c|c|c|c|}
\hline No. & Primes & $\sigma_{p}^{\nu}$ & $l$ & Num \\
\hline 1 & $\begin{array}{l}23,3767,13127,16223,18503,22247 \\
38567,39887,48647,54167, \cdots, 184727 \\
197807,204143,204983,205703 .\end{array}$ & $(0,0,1,1,1,0,1,1)$ & 38 & 198 \\
\hline ... & & & $\ldots$ & $\ldots$ \\
\hline 23 & $\begin{array}{l}\text { 479, 1151, 4919, 5519, 6599, 7559, 29399, } \\
51719,53591,67751,68879,69431,72551, \\
\text { 76919, 103319, 108191, 117431, 122471, } \\
\text { 123791, 138071, 147311, 161999, 188999, } \\
\text { 195359, 203279, 206351, 211151, 212999, } \\
217271,230999,247799,251831 .\end{array}$ & $(0,0,0,0,0,1$ & 32 & 120 \\
\hline$\ldots$ & $\ldots \ldots \ldots \ldots$ & & $\cdots$ & .. \\
\hline 47 & $1151,4919,5519,6599,7559$. & $(0,0,0,0,0,1,1,1)$ & 5 & 1 \\
\hline$\ldots$ & .. & $\cdots$ & $\ldots$ & ... \\
\hline 60 & $2503,2767,5167,5623,8887,11887,16447$. & $(0,1,1,1,0,1,0,0)$ & 7 & 4 \\
\hline
\end{tabular}

If we have $\prod_{i=1}^{6} s_{p, i} \leq Q_{11}$ in a sequence, then $p_{2}, p_{3}, p_{4}, p_{5}, p_{6}$ must be the first five primes of another sequence. There are in total three pairs of such sequences. In addition to the pair $(23,47)$ shown in Table 1 , the other pairs are $(24,56)$ and $(49,57)$. Therefore, we only need to check sequence 23,24 and 49 for $t>5$. Each one has $\prod_{i=1}^{6} s_{p, i}>Q_{11}$, so $t>5$ is impossible. For $t=5$, our algorithm runs in less than 0.1 second and finds no strong pseudoprime.

3.2. $t=4$. For $t=4$, we first define $\left(p_{1}, p_{2}, p_{3}\right)$ to be a feasible triple if it satisfies

$$
p_{1}<p_{2}<p_{3}, \quad \sigma_{p_{1}}^{\nu}=\sigma_{p_{2}}^{\nu}=\sigma_{p_{3}}^{\nu}, \quad p_{1} p_{2} p_{3}^{2}<Q_{11} .
$$

Our algorithm proceeds as follows: for each $p_{1} \leq\left[\sqrt[4]{Q_{11}}\right]=44224$, find feasible triples $\left(p_{1}, p_{2}, p_{3}\right)$. We have $\lambda_{p_{i}} \mid(n-1)$, for $i=1,2,3$. Let $\lambda=\operatorname{lcm}\left\{\lambda_{p_{1}}, \lambda_{p_{2}}, \lambda_{p_{3}}\right\}$ and $b=p_{1} p_{2} p_{3}$. Then we have

$$
n=b p_{4} \equiv 1 \quad \bmod \lambda .
$$

If $\operatorname{gcd}(b, \lambda) \neq 1$, it is impossible to have such $n$. If $\operatorname{gcd}(b, \lambda)=1$, we need to check all $p_{4}$ with

$$
p_{3}<p_{4} \leq Q_{11} / b, \quad p_{4} \equiv b^{-1} \bmod \lambda .
$$


Our algorithm takes less than 6 hours, finding 402273 feasible triples and no $\operatorname{spsp}(\nu)$ with $t=4$.

$$
\text { 4. } t=3
$$

In this section, we consider the case $t=3$. As above, we define a feasible pair $\left(p_{1}, p_{2}\right)$ by

$$
p_{1}<p_{2}, \quad \sigma_{p_{1}}^{\nu}=\sigma_{p_{2}}^{\nu}, \quad p_{1} p_{2}^{2}<Q_{11} .
$$

Our algorithm is just as in the $t=4$ case: for each $p_{1} \leq\left[\sqrt[3]{Q_{11}}\right]=1563922$, find feasible pairs $\left(p_{1}, p_{2}\right)$. Let $b=p_{1} p_{2}$ and $\lambda=\operatorname{lcm}\left(\lambda_{p_{1}}, \lambda_{p_{2}}\right)$, then $\lambda \mid(n-1)$. If $\operatorname{gcd}(b, \lambda)=1$, we check all $p_{3}$ with

$$
p_{2}<p_{3} \leq Q_{11} / b, \quad p_{3} \equiv b^{-1} \bmod \lambda .
$$

We divide our algorithm into three parts according to $p_{1} \equiv 3 \bmod 4, p_{1} \equiv 5 \bmod 8$ and $p_{1} \equiv 1 \bmod 8$.

4.1. $p_{1} \equiv 3 \bmod 4$. For $p_{1} \equiv 3 \bmod 4$, we first assume $p_{2} \equiv 3 \bmod 4$. In this case, $\sigma_{p_{1}}^{\nu}=\sigma_{p_{2}}^{\nu}$ implies $\left(\frac{a}{p_{1}}\right)=\left(\frac{a}{p_{2}}\right)$ for all $a=2,3,5,7,11,13,17,19$ by Proposition 2.3. Moreover, in this situation, the inverse is also true. We calculate the Legendre symbol $\left(\frac{a}{p_{i}}\right)$ instead of $v\left(\operatorname{ord}_{p_{i}}(a)\right)$. When searching $p_{2}$, we use the first five primes and then

$$
\left(\frac{a}{p_{1}}\right)=\left(\frac{a}{p_{2}}\right), \quad a=2,3,5,7,11,
$$

reducing to 30 residue classes modulo $9240=8 \cdot 3 \cdot 5 \cdot 7 \cdot 11$.

Example 4.1. For $p_{1}=31$, a feasible pair $\left(31, p_{2}\right)$ must satisfy

$$
p_{2} \leq\left[\sqrt{Q_{11} / 31}\right]=351270645 .
$$

If we search $p_{2}$ the same way as in the $t \leq 5$ and $t=4$ cases, we need to check all odd numbers greater than 31 ; there are about $1.7 \cdot 10^{8}$ candidates. For our method, there are only $30 \cdot \frac{351270645}{9240} \approx 1.1 \cdot 10^{6}$ candidates.

We use another trick. If $b=p_{1} p_{2}$ is less than $4 \cdot 10^{6}$, the corresponding $\lambda$ may be too small. We do not find $p_{3}$ as described above. In fact, since

$$
n=b p_{3} \equiv b \quad \bmod p_{3}-1
$$

and

$$
a^{n-1} \equiv a^{b-1} \equiv 1 \quad \bmod p_{3}, \quad a=2,3,
$$

we calculate $\operatorname{gcd}\left(2^{b-1}-1,3^{b-1}-1\right)$, and then factor it to get the prime divisors which are greater than $p_{2}$ and less than $Q_{11} / b$.

The following example explains why we need to factor the greatest common divisor instead of the usual search.

Example 4.2. Notice that for some $b$, the corresponding $\lambda$ is small. For $b=p_{1} p_{2}=$ $43 \cdot 9283=399169, \sigma_{p_{1}}^{\nu}=\sigma_{p_{2}}^{\nu}=(1,1,1,1,0,0,0,1), \lambda=9282$, we need to check all $p_{3}$ with

$$
9283<p_{3} \leq Q_{11} / b \approx 9.6 \cdot 10^{12}, \quad p_{3} \equiv 7771 \bmod 9282,
$$

and for $b=p_{1} p_{2}=571 \cdot 2851=1627921, \sigma_{p_{1}}^{\nu}=\sigma_{p_{2}}^{\nu}=(1,1,0,1,0,0,1,1), \lambda=2850$, all $p_{3}$ with

$$
2851<p_{3} \leq Q_{11} / b \approx 2.3 \cdot 10^{12}, \quad p_{3} \equiv 2281 \bmod 2850 .
$$


There are 295 feasible pairs $\left(p_{1}, p_{2}\right)$ with $p_{1} p_{2}<4 \cdot 10^{6}$ no matter whether $p_{1} \equiv 1 \bmod 4$ or $p_{1} \equiv 3 \bmod 4$. Our algorithm takes less than 8 hours, finding 21110549 feasible pairs and two $\operatorname{spsp}(\nu)$ 's:

$$
\begin{aligned}
3825123056546413051 & =149491 \cdot 747451 \cdot 34233211, \\
230245660726188031 & =214831 \cdot 787711 \cdot 1360591 .
\end{aligned}
$$

The first one is $Q_{11}$. Zhang has found these two numbers in [7] and 9].

For the case $p_{2} \equiv 1 \bmod 4$, we have $\sigma_{p_{2}}^{\nu}=\sigma_{p_{1}}^{\nu} \in\{0,1\}^{8}$. We call a prime $p \equiv$ $1 \bmod 4$ with $\sigma_{p}^{\nu} \in\{0,1\}^{8}$ a binary prime. For a prime $p \equiv 5 \bmod 8, v\left(\operatorname{ord}_{p}(2)\right)=2$, so binary primes must satisfy $p \equiv 1 \bmod 8$. We need to check binary primes $p_{2}$ with

$$
p_{1} p_{2}^{2}<Q_{11}, \quad p_{1} \geq 23 .
$$

Then $p_{2} \leq\left[\sqrt{Q_{11} / 23}\right]=407810860$. It takes 7 minutes to find a total of 51 binary primes in the range. Let $p_{2}$ be a binary prime. We check all $p_{1} \equiv 3 \bmod 4$ and $p_{1}<p_{2}$, and find 24 more feasible pairs $\left(p_{1}, p_{2}\right)$ and no $\operatorname{spsp}(\nu)$. By considering binary primes, we reduce half of the candidates at the cost of 7 minutes.

4.2. $p_{1} \equiv 5 \bmod 8$. If $p_{1} \equiv 5 \bmod 8$, we have $\left(\frac{2}{p_{1}}\right)=-1$, and then $v\left(\operatorname{ord}_{p_{1}}(2)\right)=2$. Thus for each $p_{2}$ with $\sigma_{p_{2}}^{\nu}=\sigma_{p_{1}}^{\nu}$, we must have $p_{2} \equiv 1 \bmod 4$. If $p_{2} \equiv 5 \bmod 8$, by Proposition 2.3, we use the first five primes, then

$$
\left(\frac{a}{p_{2}}\right)=\left(\frac{a}{p_{1}}\right), \quad a=2,3,5,7,11 .
$$

There are 30 residue classes module 9240 . If $p_{2} \equiv 1 \bmod 8$, by Proposition 2.4 , we must have

$$
\left(\frac{a}{p_{2}}\right)=1, \quad a=2,3,5,7,11 .
$$

There are also 30 residue classes module 9240 . As in the $p_{1} \equiv 3 \bmod 4$ case, if $p_{1} p_{2}<4 \cdot 10^{6}$, we factor $\operatorname{gcd}\left(2^{b-1}-1,3^{b-1}-1\right)$ to get a possible $p_{3}$. The total time for checking all $p_{1}$ up to 1563922 is about 11 hours and we find 1401941 feasible pairs with no $\operatorname{spsp}(\nu)$.

We give an example with $p_{1} \equiv 5 \bmod 8$ and $b<4 \cdot 10^{6}$.

Example 4.3. For $b=p_{1} p_{2}=29 \cdot 7589=220081, \sigma_{p_{1}}^{\nu}=\sigma_{p_{2}}^{\nu}=(2,2,1,0,2,1,2,2)$, $\lambda=7588$, we need to check all $p_{3}$ with

$$
7589<p_{3} \leq Q_{11} / b \approx 1.7 \cdot 10^{13}, \quad p_{3} \equiv 785 \bmod 7588 .
$$

4.3. $p_{1} \equiv 1 \bmod 8$. For $p_{1} \equiv 1 \bmod 8$, denote $e=v\left(p_{1}-1\right)$ and $f=v\left(\lambda_{p_{1}}\right)$. Then $f \leq e$ and $p_{1} \equiv 1+2^{e} \bmod 2^{e+1}$. For $p_{2}$ with $\sigma_{p_{2}}^{\nu}=\sigma_{p_{1}}^{\nu}$, we have

$$
p_{2} \equiv 1 \bmod 2^{f} \text {. }
$$

If $f=e$, we consider two cases. For $p_{2} \equiv 1+2^{e} \bmod 2^{e+1}$, by Proposition 2.3 we have

$$
\left(\frac{a}{p_{2}}\right)=\left(\frac{a}{p_{1}}\right), \quad a=2,3,5,7,11 .
$$

There are 30 residue classes module $2^{e+1} \cdot 1155$. For $p_{2} \equiv 1 \bmod 2^{e+1}$, by Proposition 2.4 , we have

$$
\left(\frac{a}{p_{2}}\right)=1, \quad a=2,3,5,7,11 .
$$


There are also 30 residue classes module $2^{e+1} \cdot 1155$. If $f<e$, we only use the condition $p_{2} \equiv 1 \bmod 2^{f}$. Our algorithm takes about 2 hours, finding 93957 feasible pairs and no $\operatorname{spsp}(\nu)$.

Until now, we finish the $t=3$ case, and find two $\operatorname{spsp}(\nu)$ 's with $p_{1} \equiv 3 \bmod 4$. The total time is about 21 hours.

$$
\text { 5. } t=2
$$

For $t=2$, there is no need to define feasible integers. If $n=p_{1} p_{2}$ is an $\operatorname{spsp}(\nu)$, as $\lambda_{p_{1}} \mid(n-1)$ and $\lambda_{p_{1}} \mid\left(p_{1}-1\right)$, we have

$$
p_{1}<p_{2} \leq Q_{11} / p_{1}, \quad p_{2} \equiv 1 \quad \bmod \lambda_{p_{1}} .
$$

Since $\lambda_{p_{1}}$ is close to $p_{1}-1$, there are about $Q_{11} /\left(p_{1}\right)^{2}$ candidates for each $p_{1}$. When $p_{1}$ is small, there are too many. According to the size of $p_{1}$, we divide our algorithm into three parts.

5.1. Small and large $p_{1}$. If $p_{1}<10^{6}$, we use the same method as for $t=3$ with $p_{1} p_{2}<4 \cdot 10^{6}$. We have

$$
a^{p_{1}-1} \equiv a^{n-1} \equiv 1 \quad \bmod p_{2}, \quad a=2,3 .
$$

We calculate $\operatorname{gcd}\left(2^{p_{1}-1}-1,3^{p_{1}-1}-1\right)$ and factor it to get prime divisors $p_{2}$ with

$$
p_{1}<p_{2} \leq Q_{11} / p_{1} .
$$

Our algorithm takes about 9 hours and finds no $\operatorname{spsp}(\nu)$.

For $p_{1}>10^{8}$, almost all $p_{1}$ with $\lambda_{p_{1}}=p_{1}-1$. There are less than $Q_{11} / 10^{16} \approx 380$ candidates. We just run the algorithm as described at the beginning of this section. It takes about 19 hours and finds five $\operatorname{spsp}(\nu)$ 's:

$$
\begin{aligned}
84983557412237221 & =206135341 \cdot 412270681, \\
1134931906634489281 & =753303361 \cdot 1506606721, \\
1144336081150073701 & =756417901 \cdot 1512835801, \\
1167748053436849501 & =764116501 \cdot 1528233001, \\
1646697619851137101 & =907385701 \cdot 1814771401 .
\end{aligned}
$$

5.2. $10^{6}<p_{1}<10^{8}$. When $p_{1}$ is in this interval, $p_{1}$ is too large and calculating $2^{p_{1}-1}-1$ and $3^{p_{1}-1}-1$ takes too much time. It is also too small and there are too many candidates for $p_{2}$ with

$$
p_{1}<p_{2} \leq Q_{11} / p_{1}, \quad p_{2} \equiv 1 \quad \bmod \lambda_{p_{1}} .
$$

We need to use the Chinese Remainder Theorem to reduce the number of candidates. Just as in the $t=3$ case, we divide the algorithm into three parts according to $p_{1} \equiv 3 \bmod 4, p_{1} \equiv 5 \bmod 8$ and $p_{1} \equiv 1 \bmod 8$. The difference is that we use the first six primes in these cases.

For $p_{1} \equiv 3 \bmod 4$, we find $p_{2}$ with $\sigma_{p_{2}}^{\nu}=\sigma_{p_{1}}^{\nu}$. If $p_{2} \equiv 3 \bmod 4$, then by Proposition 2.3

$$
\left(\frac{a}{p_{1}}\right)=\left(\frac{a}{p_{2}}\right), \quad a=2,3,5,7,11,13 .
$$

If $p_{2} \equiv 1 \bmod 4$, then by Proposition 2.4

$$
\left(\frac{a}{p_{2}}\right)=1, \quad a=2,3,5,7,11,13 .
$$


Since $8 \cdot 3 \cdot 5 \cdot 7 \cdot 11 \cdot 13=120120$, together with $p_{2} \equiv 1 \bmod \lambda_{p_{1}}$, in each case there are at most 180 residue classes modulo $\operatorname{lcm}\left(\lambda_{p_{1}}, 120120\right)$. Our algorithm takes about 16 hours and finds no $\operatorname{spsp}(\nu)$.

If $p_{1} \equiv 5 \bmod 8$, then $p_{2} \equiv 1 \bmod 4$. If $p_{2} \equiv 5 \bmod 8$, then we have $p_{2} \equiv$ $1 \bmod \lambda_{p_{1}}$ and

$$
\left(\frac{a}{p_{1}}\right)=\left(\frac{a}{p_{2}}\right), \quad a=2,3,5,7,11,13 .
$$

If $p_{2} \equiv 1 \bmod 8$, then we have $p_{2} \equiv 1 \bmod \lambda_{p_{1}}$ and

$$
\left(\frac{a}{p_{2}}\right)=1, \quad a=2,3,5,7,11,13 .
$$

Our algorithm takes about 15 hours and finds only one $\operatorname{spsp}(\nu)$ :

$$
341550071728321=10670053 \cdot 32010157,
$$

which is $\psi_{8}$.

For $p_{1} \equiv 1 \bmod 8$, denote $e=v\left(p_{1}-1\right)$ and $f=v\left(\lambda_{p_{1}}\right)$. Then $f \leq e$. If $f=e$, there are two cases. For $p_{2} \equiv 1+2^{e} \bmod 2^{e+1}$, then $p_{2} \equiv 1 \bmod \lambda_{p_{1}}$ and

$$
\left(\frac{a}{p_{1}}\right)=\left(\frac{a}{p_{2}}\right), \quad a=2,3,5,7,11,13 .
$$

If $p_{2} \equiv 1 \bmod 2^{e+1}$, then $p_{2} \equiv 1 \bmod \lambda_{p_{1}}$ and

$$
\left(\frac{a}{p_{2}}\right)=1, \quad a=2,3,5,7,11,13 .
$$

If $f<e$, we only use the condition $p_{2} \equiv 1 \bmod \lambda_{p_{1}}$, Our algorithm takes about 18 hours and finds no $\operatorname{spsp}(\nu)$.

Then we finish the $t=2$ case and find six strong pseudoprimes to the first eight prime bases. It takes 77 hours.

\section{Conclusion}

Until now, we have checked all the odd composite numbers up to $Q_{11}$, and find eight strong pseudoprimes to the first eight prime bases. See Table 2 .

TABLE 2. Spsp's $n \leq Q_{11}$ to the first eight prime bases

\begin{tabular}{|l|r|l|}
\hline & Number & Factorization \\
\hline \multirow{3}{*}{$t=3$} & 3825123056546413051 & $149491 \cdot 747451 \cdot 34233211$ \\
\cline { 2 - 3 } & 230245660726188031 & $214831 \cdot 787711 \cdot 1360591$ \\
\hline \multirow{4}{*}{$t=2$} & 84983557412237221 & $206135341 \cdot 412270681$ \\
\cline { 2 - 3 } & 1134931906634489281 & $753303361 \cdot 1506606721$ \\
\cline { 2 - 3 } & 1144336081150073701 & $756417901 \cdot 1512835801$ \\
\cline { 2 - 3 } & 1167748053436849501 & $764116501 \cdot 1528233001$ \\
\cline { 2 - 3 } & 1646697619851137101 & $907385701 \cdot 1814771401$ \\
\cline { 2 - 3 } & 341550071728321 & $10670053 \cdot 32010157$ \\
\hline
\end{tabular}

The first row is $Q_{11}$ and the last row is $\psi_{8}$. It is easy to check that $Q_{11}$ is also a strong pseudoprime to the bases 23, 29 and 31. The other seven numbers are not pseudoprimes to the base 23. Therefore, we have Theorem 1.1:

$$
\psi_{9}=\psi_{10}=\psi_{11}=3825123056546413051 .
$$


Thus, for an integer less than $Q_{11}$, only nine strong pseudoprime tests are needed to judge its primality and compositeness. We use Magma and all algorithms are run on a PC (an Intel(R) Core(TM)2 Duo CPU E7500 at $2.93 \mathrm{GHz}$ with $2 \mathrm{~Gb}$ of RAM). The total time is about 104 hours.

Our algorithm is similar to Jaeschke's [2, but there are some differences. In the $t=3$ case, when feasible pairs $\left(p_{1}, p_{2}\right)$ with $b=p_{1} p_{2}<4 \cdot 10^{6}$, we factor $\operatorname{gcd}\left(2^{b-1}-1,3^{b-1}-1\right)$ to get $p_{3}$ instead of searching $p_{3}$ in the range

$$
p_{2}<p_{3} \leq Q_{11} / b, \quad p_{3} \equiv b^{-1} \bmod \lambda .
$$

In contrast, Jaeschke just searched $p_{3}$ directly. This is necessary in our case as we search $p_{3}$ for the first feasible pair given in Example 4.2, i.e., $p_{1}=43, p_{2}=9283$. It requires more than 4 hours, but factorization only needs seconds. In the $t=2$ case, Jaeschke did not use the Chinese Remainder Theorem and searched $p_{2}$ in the range

$$
p_{1}<p_{2} \leq Q_{11} / p_{1}, \quad p_{2} \equiv 1 \quad \bmod \lambda_{p_{1}} .
$$

Our first version algorithm did the same thing. As our upper bound $Q_{11}$ is much greater than his $\psi_{8}$, it took more than 10 days. By Proposition 2.3 and Proposition 2.4 , we can use the Chinese Remainder Theorem to find $p_{2}$ with $\sigma_{p_{2}}=\sigma_{p_{1}}$ except for the case

Such primes are rare.

$$
p_{1} \equiv 1 \quad \bmod 8, \quad v\left(\lambda_{p_{1}}\right)<v\left(p_{1}-1\right) .
$$

For future work, Zhang has conjectured in [7] that

$$
\begin{aligned}
\psi_{12}=N_{12} & =318665857834031151167461(24 \text { digits }) \\
& =399165290221 \cdot 798330580441,
\end{aligned}
$$

which is the smallest known strong pseudoprime to the first 12 prime bases. We need to check all odd integers up to this bound to determine the exact value of $\psi_{12}$. Only for the $t=2$ case, do we have to search $p_{2}$ for all $p_{1} \leq \sqrt{N_{12}} \approx 5.6 \cdot 10^{11}$. It is beyond the capability of a personal computer even if we use the Chinese Remainder Theorem.

We should mention Charles Greathouse's computation. He also reached the conclusion that $\psi_{9}=\psi_{10}=\psi_{11}=Q_{11}$ based on the output of Jan Feitsma, who computed all odd psp(2)'s up to $2^{64}$. Feitsma's result is available at http:// www.cecm.sfu.ca/Pseudoprimes/index-2-to-64.html and Greathouse's result is stated as A014233 in OEIS. Our calculation is independent of their results and it is more efficient.

\section{ACKNowledgments}

The authors thank the anonymous referees for many suggestions on how to improve the presentation of this paper.

\section{REFERENCES}

[1] François G. Dorais and Dominic Klyve, A Wieferich prime search up to $6.7 \times 10^{15}$, J. Integer Seq. 14 (2011), no. 9, Article 11.9.2, 14. MR2859986

[2] Gerhard Jaeschke, On strong pseudoprimes to several bases, Math. Comp. 61 (1993), no. 204, 915-926, DOI 10.2307/2153262. MR1192971 (94d:11004)

[3] Joshua Knauer and Jörg Richstein, The continuing search for Wieferich primes, Math. Comp. 74 (2005), no. 251, 1559-1563 (electronic), DOI 10.1090/S0025-5718-05-01723-0. MR2137018 (2006a:11006) 
[4] Carl Pomerance, J. L. Selfridge, and Samuel S. Wagstaff Jr., The pseudoprimes to $25 \cdot 10^{9}$, Math. Comp. 35 (1980), no. 151, 1003-1026, DOI 10.2307/2006210. MR572872 (82g:10030)

[5] Michael O. Rabin, Probabilistic algorithm for testing primality, J. Number Theory 12 (1980), no. 1, 128-138, DOI 10.1016/0022-314X(80)90084-0. MR566880(81f:10003)

[6] Zhenxiang Zhang, Finding strong pseudoprimes to several bases, Math. Comp. 70 (2001), no. 234, 863-872, DOI 10.1090/S0025-5718-00-01215-1. MR.1697654 (2001g:11009)

[7] Zhenxiang Zhang, Finding $C_{3}$-strong pseudoprimes, Math. Comp. 74 (2005), no. 250, 1009 1024 (electronic), DOI 10.1090/S0025-5718-04-01693-X. MR2114662 (2005k:11243)

[8] Zhenxiang Zhang, Two kinds of strong pseudoprimes up to $10^{36}$, Math. Comp. 76 (2007), no. 260, 2095-2107 (electronic), DOI 10.1090/S0025-5718-07-01977-1. MR2336285 (2008h:11114)

[9] Zhenxiang Zhang and Min Tang, Finding strong pseudoprimes to several bases. II, Math. Comp. 72 (2003), no. 244, 2085-2097 (electronic), DOI 10.1090/S0025-5718-03-01545-X. MR1986825 (2004c:11008)

Key Laboratory of Mathematics Mechanization, NCMis, Academy of Mathematics And Systems Science, Chinese Academy of Sciences, Beijing, China, 100190

E-mail address: jiangyupeng@amss.ac.cn

Key Laboratory of Mathematics Mechanization, NCMis, Academy of Mathematics and Systems Science, Chinese Academy of Sciences, Beijing, China, 100190

E-mail address: dengyp@amss.ac.cn 